Published in final edited form as:

J Am Geriatr Soc. 2017 February ; 65(2): 373-380. doi:10.1111/jgs.14368.

\title{
Afternoon Napping and Cognition in Chinese Older Adults: Findings from the China Health and Retirement Longitudinal Study Baseline Assessment
}

\author{
Junxin Li, PhD ${ }^{\star}, \dagger$, Pamela Z. Cacchione, $\mathrm{PhD}^{\dagger}$, Nancy Hodgson, $\mathrm{PhD}^{\dagger}$, Barbara Riegel, \\ $\mathrm{PhD}^{\dagger}$, Brendan T. Keenan, MS ${ }^{\star}$, Mathew T. Scharf, MD, PhD*, Kathy C. Richards, $\mathrm{PhD}^{\ddagger}$, and \\ Nalaka S. Gooneratne, MD ${ }^{*}, \S$
}

*Center for Sleep and Circadian Neurobiology †School of Nursing, Johns Hopkins University, Baltimore, Maryland $¥$ School of Nursing, College of Health and Human Services, George Mason University, Fairfax, Virginia §Division of Geriatric Medicine, School of Medicine, University of Pennsylvania, Philadelphia, Pennsylvania.

\section{Abstract}

OBJECTIVES: To examine the cross-sectional associations between self-reported postlunch napping and structured cognitive assessments in Chinese older adults.

DESIGN: Cross-sectional cohort study.

SETTING: China.

PARTICIPANTS: Individuals aged 65 and older from the baseline national wave of the China Health and Retirement Longitudinal Study (CHARLS) $(\mathrm{N}=2,974)$.

MEASUREMENTS: Interview-based cognitive assessments of orientation and attention, episodic memory, visuospatial abilities, and a combined global cognition score incorporating these assessments. Other self-reported or interview-based assessments included postlunch napping duration, nighttime sleep duration, demographic characteristics, health habits, comorbidities, functional status and social activities. According to reported napping duration, older adults were categorized as non-nappers ( 0 minutes), short nappers ( $<30$ minutes), moderate nappers (30-90 minutes), and extended nappers (>90 minutes).

RESULTS: Postlunch napping was reporting in 57.7\% of participants for a mean of 63 minutes. Cognitive function was significantly associated with napping $(P<.001)$. Between-group comparisons showed that moderate nappers had better overall cognition than non-nappers $(P<$. $001)$ or extended nappers $(P=.01)$. Non-nappers also had significantly poorer cognition than short

Address correspondence to Junxin Li, 3624 Market Street, Suite 201, Philadelphia, PA 19104. junxin.li@ uphs.upenn.edu. Author Contributions: All authors contribute to: the conception and design of this study; analysis and interpretation of data; drafting and revising the article; and final approval of the version to be published.

Conflict of Interest: The authors declare no competing interests.

SUPPORTING INFORMATION

Additional Supporting Information may be found in the online version of this article:

Please note: Wiley-Blackwell is not responsible for the content, accuracy, errors, or functionality of any supporting materials supplied by the authors. Any queries (other than missing material) should be directed to the corresponding author for the article. 
nappers $(P=.03)$. In multiple regression analysis, moderate napping was significantly associated with better cognition than non- $(P=.004)$, short $(P=.04)$, and extended napping $(P=.002)$, after controlling for demographic characteristics, body mass index, depression, instrumental activities of daily living, social activities, and nighttime sleep duration.

CONCLUSION: A cross-sectional association was found between moderate postlunch napping and better cognition in Chinese older adults. The cross-sectional design and self-reported measures of sleep limited the findings. Longitudinal studies with objective napping measures are needed to further test this hypothesis.

\section{Keywords}

postlunch napping; sleep duration; cognition; older adults

Cognitive impairment significantly increases the risk of functional dependence and poor quality of life in older adults. ${ }^{1,2}$ Current research has focused on the prevention or delay of cognitive decline through risk factor identification and modification, with growing interest in the role of sleep behaviors, such as the optimal duration of sleep and napping. ${ }^{3-5}$ The prevalence of habitual daytime napping ranges from $22 \%$ to $69 \%$ in older adults around the world, which is significantly higher than in younger individuals. ${ }^{6-9}$ Habitual afternoon napping, such as routinely taking a nap after lunch, is prevalent in Chinese older adults and is considered part of a healthy lifestyle from a cultural perspective. .,10 $^{3}$

Prior research has investigated the short-term positive and negative effects of different napping durations on cognition, ${ }^{11-18}$ but the association between napping duration and longterm cognitive function in older adults remains poorly understood, and the results in the literature are inconsistent. A few studies have reported that longer napping duration is associated with cognitive impairment, ${ }^{3-5}$ but other studies have found beneficial effects of napping on cognitive function. ${ }_{19}$ Daytime napping may also be related to negative nighttime sleep outcomes, such as shorter sleep duration and frequent nocturnal awakening. ${ }^{8,20}$

The circadian timing and duration of daytime napping may enhance the likelihood of beneficial effects from napping. ${ }^{21}$ The postlunch circadian dip period (1:00-4:00 p.m.) is the most common and preferred time for naps. ${ }^{11,12,21}$ Naps during this period are more restorative than naps in the early morning and evening; for example, evening naps taken during the circadian nadir generated less-restorative outcomes than naps taken at other times. 11,21

According to a review of studies on napping and cognition, short-term positive and negative effects on cognition were shown with different nap durations. ${ }^{21}$ The short-term benefits of brief naps (e.g., 10 minutes) are well documented in previous studies and include greater alertness and accuracy and speed when performing a number of cognitive tasks, including psychomotor performance and short-term memory, ${ }^{13-16,21}$ although longer naps (e.g., >30 minutes) may lead to a transient short-term deterioration in cognitive performance from sleep inertia followed by a period of improved cognitive function for up to 24 hours. ${ }^{17,21}$ Furthermore, long nap durations during the day may disturb nighttime sleep, affect circadian 
rhythms, interfere with daytime engagements, and potentiate sleep inertia-related cognitive impairments. ${ }^{8,20}$

In the current study, it was hypothesized that moderate-duration naps taken during the postlunch dip would be associated with better cognitive performance in Chinese older adults and that extended nap duration would be associated with shorter nighttime sleep duration. To assess these hypotheses, cross-sectional associations between self-reported postlunch napping duration and cognitive function were examined using baseline data from the China Health and Retirement Longitudinal Study (CHARLS). Characteristics of older adults were evaluated according to different durations of postlunch napping, and the association between postlunch napping and nighttime sleep duration was also evaluated. The findings from this study are particularly relevant given the aging demographic patterns of China, which has 200 million adults aged 60 and older, estimated to increase to 400 million by $2050 .^{22}$

\section{METHODS}

\section{Study Population}

This was a retrospective, cross-sectional study conducted using the CHARLS baseline dataset. CHARLS is a nationally representative longitudinal study of Chinese communitydwelling residents aged 45 and older and their spouses. It was designed based on the Health and Retirement Study (HRS) in the United States, with the aim of serving the needs of scientific research related to the rapid aging of the population in China and supporting scientific research in middle-aged and older adults. ${ }^{23}$ The CHARLS baseline dataset consists of 17,708 respondents evaluated between June 2011 and March 2012. ${ }^{23}$ CHARLS offers a wide range of information on socioeconomic status and health, including demographic characteristics, family structure, health status and functioning, biomarkers, health care and insurance, work, retirement and pension, income and consumption, assets (individual and household), and community-level information. ${ }^{24}$

To be included in the current analysis, respondents needed to be aged 65 and older and have complete data on age, sex, education, body mass index (BMI), postlunch napping duration, and cognition measures. Four thousand seven hundred seventy-six respondents in this cohort (27\%) were eligible for inclusion, after restricting to participants aged 65 and older. Of these, 1,249 (26.2\%) were excluded because postlunch napping duration or cognition measures were missing and $553(11.6 \%)$ because information on BMI was missing, leaving $2,974(62.3 \%)$ respondents with complete data in the relevant domains for the analyses presented herein.

\section{Measures in CHARLS}

\section{Cognitive Assessment}

The cognitive dimensions of orientation, attention, episodic memory, and visuospatial abilities were assessed. Consistent with prior CHARLS publications, four cognition scores were used: items from the Telephone Interview of Cognitive Status (TICS-10), word recall, figure drawing, and an overall cognition score incorporating these assessments. ${ }^{25}$ Overall cognition score was considered to be the primary outcome of interest. The relationship 
between postlunch nap duration and the three domain specific measures were examined in secondary analyses. The overall cognition score represented respondents' cognitive status and is the sum score of the TICS-10 (orientation and attention), word recall (episodic memory), and figure drawing (visual spatial abilities), which ranged from 0 to 21 . The individual measures are described in more detail below.

Telephone Interview of Cognitive Status.-The TICS is a well-established measure of an individual's mental status. ${ }^{26}$ Ten mental status questions from the TICS were used in CHARLS: serial subtraction of 7 from 100 (up to five times), date (month, day, year), day of the week, and season of the year. The TICS-10 score was the aggregate number of correct answers and ranged from 0 to $10 .^{25,26}$ TICS-10 primarily measures the executive functions of orientation to time and attention. ${ }^{27,28}$

Word Recall.—Respondents were asked to memorize and immediately recall as many words as they could in any order after interviewers read a list of 10 Chinese nouns (immediate recall). Four to 10 minutes later, respondents were asked to recall as many of the original words as possible (delayed recall). ${ }^{25,26}$ An episodic memory measure was created according to previous recommendations. ${ }^{29}$ The episodic memory score consisted of the average number of immediate and delayed word recalls and ranged from 0 to $10 .{ }^{29}$

Figure Drawing.-Respondents were shown a picture of two overlapped pentagons and asked to draw a similar figure. "Respondents who successfully completed the task received a score of 1 , and those who failed received a score of $0 .{ }^{25}$ This task assessed visuospatial abilities. ${ }^{30}$

\section{Sleep Variables}

Two self-reported variables related to sleep habits were studied: postlunch napping duration and total nighttime sleep duration.

Postlunch Napping Duration.-Postlunch napping duration was assessed by asking, "During the past month, how long did you take a nap after lunch in general?" Respondents were categorized into four napping groups: non-nappers ( 0 minutes), short nappers $(<30$ minutes), moderate nappers (30-90 minutes), and extended nappers ( $>90$ minutes). The cut points for napping groups were chosen after reviewing the literature; 30 minutes is a commonly used cutoff for short napping, ${ }^{7,29}$ and 90 minutes is an advised practice maximum for naps. ${ }^{20}$

Nighttime Sleep Duration.-Nighttime sleep duration was assessed by asking, "During the past month, how many hours of actual sleep did you get at night (average hours for one night)? (This may be shorter than the number of hours you spend in bed.)." Respondents were classified into four nighttime sleep groups: very short sleeper ( $<5$ hours/night), short sleeper (5-7 hours/night), normal sleeper (7-9 hours/night), and long sleeper ( $\geq 9$ hours/ night). These cut points were chosen according to the existing epidemiological literature on sleep duration. ${ }^{31,32}$ 


\section{Covariates}

Demographic characteristics, health habits, self-reported comorbidity, functional status, and social activities were considered as covariates in this study.

Demographic characteristics included age, sex, education, and marital status. Consistent with prior CHARLS publications, education was categorized into four groups: illiterate, some primary school (can read or write), finished primary school, and junior high or above. 25,33 Marital status was grouped as married versus not married.

Health habits included body mass index (BMI; $\mathrm{kg} / \mathrm{m}^{2}$ ), smoking, and drinking. Respondents were categorized as nonsmokers, light or moderate smokers $(<20$ cigarettes per day currently or history of smoking), and heavy smoker ( $\geq 20$ cigarettes per day currently). ${ }^{34}$ Respondents were categorized as nondrinkers, mild or moderate drinkers (currently drinking less than twice a day), and heavy drinkers (currently drinking more than twice a day). ${ }^{34}$

Self-reported comorbidity included self-reported chronic disease diagnoses and medications for treating those diseases and was assessed as the total number of self-reported chronic disease diagnoses. To provide additional information regarding common diseases in older adults, whether the respondent had hypertension or cardiovascular disease was separately included. Depression was measured using the 10-item Center for Epidemiologic Studies Depression Scale (CES-D-10), with depression defined as a score of 10 or greater. $^{34}$

Functional status was measured according to activity of daily living (ADLs) disability (range 0-6) and instrumental activities of daily living (IADLs) (range 0-5). ${ }^{35}$ Social activities were assessed by asking whether the respondent participated in specific activities such as interacting with friends or assisting others ${ }^{23}$ and categorized as almost no social activity $(<1 /$ week), some social activity (1/week), and socially active (>1/week).

\section{Statistical Analysis}

Characteristics of the overall sample and according to napping group were described using means and standard deviations for continuous data and percentages for categorical data. Differences between napping groups were examined using analysis of variance for normally distributed variables, the nonparametric Kruskal-Wallis test for skewed continuous variables, and the chi-square test for categorical variables. Adjusted analyses were performed using linear or logistic regression models with napping groups as a predictor variable. The distributions of overall cognition, TICS-10, and word recall were checked and resulted in normal distribution, allowing for parametric analysis on untransformed variables. Results of linear regression analyses are presented using b-coefficients and $95 \%$ confidence intervals (95\% CIs). Logistic regression results are presented using odds ratios (ORs) and 95\% CIs. If there were significant overall differences of cognition measures and night sleep duration between the napping groups, pairwise comparisons were performed between napping groups using a Tukey-Kramer adjustment for multiple comparisons in post hoc analyses.

Factors known to be associated with cognitive function were examined for inclusion in the regression models as possible confounders, with variables found to be associated with 
overall cognition in bivariate analyses at $P<.10$ retained in regression models. Model 1 (demographic factors) was adjusted for age, sex, education, and marital status. Model 2 (demographic plus medical and social) included model 1 covariates plus BMI, cardiovascular disease, depression, number of medications, drinking, smoking, ADLs, IADLs, social activity, and nighttime sleep duration. All covariates found to be significantly associated $(P<.05)$ with overall cognition in Model 2 were included in the final model (Model 3).

As discussed above, the overall cognition score was the primary outcome. Therefore, statistical significance for associations with overall cognition was based on $P<.05$. In complementary analyses, associations with the three individual submeasures of cognition included in the overall cognition score were examined, with significance based on a Bonferroni corrected $P<.0167$ (.05/3). Significance in all other analyses was based on a two-tailed $P<.05$.

\section{RESULTS}

\section{Sample Characteristics}

After applying the inclusion criteria, 2,974 older adults were included in the analytical sample. The mean age of the sample was $71.4 \pm 5.4$, and $48.7 \%$ were female. One thousand seven hundred seventeen $(57.7 \%)$ cohort members reported taking a postlunch nap, with a mean napping duration of $62.9 \pm 41.8$ minutes. This cohort reported a mean of $6.2 \pm 2.1$ hours of nighttime sleep. Detailed characteristics of overall sample and according to postlunch napping groups are presented in Table 1.

\section{Differences in Postlunch Napping Groups}

The differences in sample characteristics between napping groups are presented in Table 1. There were significant differences $(P<.05)$ between groups with respect to sex, marital status, education, smoking, drinking, BMI, number of reported medications, number of comorbid diseases, depression, hypertension, cardiovascular disease, and IADLs.

\section{Association Between Postlunch Napping and Nighttime Sleep Duration}

The associations between postlunch napping and nighttime sleep are presented in Table 2 and Figure 1A. Postlunch napping was significantly associated with nighttime sleep when measured as reported hours $(P<.001)$ or categorized into nighttime sleep groups $(P<.001)$. Overall, participants who reported less napping tended to have less nighttime sleep duration. Between-napping group comparisons showed that non-nappers slept significantly less than the other three groups (all $P \leq .005$ ). Extended nappers had significantly longer nighttime sleep than moderate nappers $(P=.01)$.

\section{Associations Between Possible Predictors and Cognition Measures}

The association between possible predictors and cognition measures are presented in Table $\mathrm{S} 1$. Older age, female sex, not being married, less education, depression, lower BMI, and greater ADL and IADL dependency were significantly associated with worse overall cognition and each cognitive assessment separately. Cardiovascular disease was significantly 
associated with worse cognition (overall cognition, word recall, figure drawing).

Respondents who were socially active had better cognition (overall cognition, TICS-10, word recall) than those who participated in some weekly social activities, and those who reported no social activity had worse cognition according to all four measures. Drinking alcohol was positively associated with overall cognition and TICS- 10 .

\section{Postlunch Napping and Cognition Measures}

All four cognition measures (overall cognition, TICS-10, word recall, figure drawing) were significantly associated with postlunch napping groups (Table 2). Between-group comparisons showed that moderate nappers had significantly higher overall cognitive scores than non-nappers $(P<.001)$ and extended nappers $(P=.01)$. Short nappers also had significantly higher overall cognition scores than non-nappers $(P=.03)$. Moderate nappers had significantly higher TICS-10 scores than non-nappers $(P<.001)$ and extended nappers $(P=.02)$. Word recall of moderate nappers was significantly higher than that of non-nappers $(P=.001)$.

In the multiple linear regression analyses (Table 3 ), postlunch napping was significantly associated with overall cognition $(P<.001)$ and TICS-10 $(P<.001)$ in the partially adjusted model, which was adjusted for demographic characteristics including age, sex, education, and marital status (Model 1); the associations remained significant in the fully adjusted models that adjusted for all covariates including demographic characteristics, health habits (BMI, smoking, drinking), self-reported comorbidity (cardiovascular disease, depression, number of disease, self-reported medication use), functional status (ADLs, IADLs), social activity, and nighttime sleep (Model 2). In the final model (Model 3), which was adjusted for significant covariates in Model 2, napping groups were independently associated with overall cognition $(P=.003)$ and TICS-10 $(P=.008)$. The adjusted 95\% CIs of mean overall cognition according to napping groups from Model 3 are presented in Figure 1B. Nonnappers had a 0.40 -point lower overall cognition score than moderate nappers, short nappers had a 0.44-point lower score, and extended nappers had a 0.59-point lower score. In Model 3 , a 1-year increase in age was associated with a 0.09 -unit drop in overall cognition $(\beta=$ $0.09,95 \% \mathrm{CI}=0.11-0.07, P<.001)$. Therefore, the differences in cognition between nap groups (non-nappers $(0.40)$, short-nappers $(0.44)$, extended nappers $(0.59)$ vs moderatenappers) were approximately four to six times as great as the association with age. Therefore, associations with napping were similar (or greater to) the change in cognition expected for a 5-year increase in age (0.45). In addition, extended napping was significantly associated with a 0.42 -point lower TICS-10 score than moderate napping. For word recall (episodic memory), the associations between napping groups and word recall were no longer significant in Model 3. No significant associations between figure drawing (visuospatial abilities) and napping group were found in any of the three models.

\section{DISCUSSION}

In this cross-sectional analysis, the association between self-reported postlunch napping duration and cognition was examined in a nationally representative sample of 2,974 community-dwelling Chinese older adults. The results support the hypothesis that a 
moderate-duration nap taken during the postlunch dip is associated with better overall cognition. Older adults who did not nap or napped longer than 90 minutes (extended nappers) were significantly more likely than those who napped for 30 to 90 minutes after lunch (moderate nappers) to have lower overall cognition scores after adjusting for possible confounders. The negative association between nap duration and nighttime sleep duration that was hypothesized was not supported in the analysis: extended nappers slept significantly longer than non-nappers and moderate nappers. Non-nappers slept the least at night of the napping groups.

A number of factors, such as the timing, duration, frequency, and planned or unplanned nature of naps, need to be considered when determining the benefit of daytime napping. ${ }^{21}$ The timing of self-reported napping in this study was considered to be in the postlunch dip period, beause the nap question was, "How long did you take a nap after lunch in general." Napping during the postlunch dip period may lead to better cognitive function because postlunch dip napping has greater recuperative value than napping at other times of the day. 21

Most prior studies on sleep and cognition in older adults focused on nighttime sleep rather than daytime napping. ${ }^{3,36,37}$ Even in studies that examined daytime sleep and cognition in older adults, napping was not assessed as a primary variable. ${ }^{17,38}$ Most studies categorized the study sample into two groups, such as nappers versus non-nappers or napping less than 2 hours versus longer than 2 hours. ${ }^{3,4}$ There was thus a need for studies to examine the association between cognition and naps of different duration. Therefore, the durations of napping in the present study were categorized into four napping groups. The inverted Ushaped association seen between cognition and nap duration presented here highlights the importance of this approach. This study suggests that absence of napping and too much napping are associated with poorer cognition, but naps of a moderate duration are associated with better cognition and may be an important part of optimizing cognition in elderly adults.

In the final analysis, no napping, short napping, and extended napping were associated with worse overall cognition than moderate napping. The difference in overall cognition associated with these napping groups was similar to or greater than the decline in cognition associated with a 5-year increase in age. This parallels findings in the literature. For example, an epidemiological study in older women showed that objectively measured (actigraphy) napping for longer than 2 hours, as determined using actigraphy, was associated with greater risk of cognitive impairment. ${ }^{36}$ Prior studies also found that extended napping was associated with worse cognition. ${ }^{4} \mathrm{~A}$ longitudinal study found that napping for less than 1 hour and longer than 1 hour were negatively associated with incidence of cognitive impairment at 2-year follow up after adjusting for multiple confounders. Napping for longer than 1 hour was also adversely associated with cognitive decline at 10 -year follow-up. ${ }^{19}$ Napping duration measured in all the studies mentioned above represents the total nap duration throughout the day, rather than napping at a certain time of the day, as was measured in the current study.

The current study found that non-nappers reported the shortest nighttime sleep duration, and extended nappers slept more during the night than non-nappers and moderate nappers. This 
finding is inconsistent with a previous study that found daytime napping to be associated with short nighttime sleep duration. ${ }^{20}$ One possible explanation for this finding is that people who did not take naps in the afternoon were genetic short sleepers and therefore also reported short nighttime sleep duration. Another explanation is that the nighttime sleep of extended nappers may be highly fragmented and their sleep efficiency may be lower than that of other groups, so they may spend more time napping to compensate for disturbed nighttime sleep, but information on sleep fragmentation and sleep efficiency was not available for analysis in this dataset. In addition, inherent limitations in using self-reported measures may have biased these results, because participants may consistently under-or overestimate their napping and nocturnal sleep duration.

This study had several additional limitations. It was cross-sectional, so no causal associations between postlunch nap duration and cognition can be drawn from the analysis. Hence, whether the duration of napping resulted in the cognitive differences or the differences in cognition led to varied nap durations could not be determined. In addition, the self-reported measure of postlunch napping may be imprecise, introducing bias to the analysis and leading to inaccurate results. Napping duration measured objectively may be beneficial in future research. Moreover, detailed information on frequency and intention of napping is not available in CHARLS (intentional vs unintentional). The majority of existing epidemiological studies do not distinguish intentional from unintentional naps in their analyses. According to the nap question asked in this study, it was assumed that the selfreported postlunch naps were intentional naps and were taken routinely. Exact information on napping duration at times other than the postlunch dip period was not available. Finally, the overall cognition score mainly represented orientation and attention (TICS-10) and episodic memory (word recall), with figure drawing contributing only 0 or 1 point. Thus, the ability to examine degree of visuospatial deficits was limited, although this scoring approach is similar to that used in the Mini-Mental State Examination. ${ }^{39}$

Despite these limitations, this study has several strengths. First, a nationally representative cohort of community-dwelling Chinese older adults, which provides a good representation of the study population, was used. Second, the robustness of the CHARLS survey allowed the independent association between postlunch napping and cognition to be studied by adjusting for multiple possible confounders.

\section{Supplementary Material}

Refer to Web version on PubMed Central for supplementary material.

\section{ACKNOWLEDGMENTS}

Financial Disclosure: This study was supported by an NIH nursing/neuroscience training grant at the University of Pennsylvania (T32 HL07953). This study is based on the baseline of the China Health and Retirement Longitudinal Study (CHARLS). We would like to thank the CHARLS research team, the field team, and every respondent for their time and efforts that they have devoted to the CHARLS project.

Sponsor's Role: None. 


\section{REFERENCES}

1. Birren JE, Lubben JE, Rowe JC et al. eds. The Concept and Measurement of Quality of Life in the Frail Elderly San Diego, CA: Academic Press, 2014.

2. Bolandzadeh N, Kording K, Salowitz N et al. Predicting cognitive function from clinical measures of physical function and health status in older adults. PLoS ONE 2015;10:e0119075. [PubMed: 25734446]

3. Blackwell T, Yaffe K, Ancoli-Israel S et al. Association of sleep characteristics and cognition in older community-dwelling men: The MrOS Sleep Study. Sleep 2011;34:1347-1356. [PubMed: 21966066]

4. Li J, Chang YP, Porock D. Factors associated with daytime sleep in nursing home residents. Res Aging 2015;37:103-117. [PubMed: 25651553]

5. Picarsic JL, Glynn NW, Taylor C et al. Self-reported napping and duration and quality of sleep in the Lifestyle Interventions and Independence for Elders pilot study. J Am Geriatr Soc 2008;56:16741680. [PubMed: 18662202]

6. dos Santos AA, Ceolim MF, Iost Pavarini SC et al. Association between sleep disorders and frailty status among elderly. Acta Paulista Enfermagem 2014;27:120-125.

7. Fang W, Li Z, Wu L et al. Longer habitual afternoon napping is associated with a higher risk for impaired fasting plasma glucose and diabetes mellitus in older adults: Results from the DongfengTongji cohort of retired workers. Sleep Med 2013;14:950-954. [PubMed: 23831240]

8. Martin JL, Ancoli-Israel S. Napping in older adults. Sleep Med Clin 2006;1:177-186.

9. Pace-Schott EF, Spencer RM. Age-related changes in the cognitive function of sleep. Prog Brain Res 2011;191:75-89. [PubMed: 21741545]

10. Cao Z, Shen L, Wu J et al. The effects of midday nap duration on the risk of hypertension in a middle-aged and older Chinese population: A preliminary evidence from the Tongji-Dongfeng Cohort Study, China. J Hypertens 2014;32:1993-1998. [PubMed: 25023156]

11. Milner CE, Cote KA. Benefits of napping in healthy adults: Impact of nap length, time of day, age, and experience with napping. J Sleep Res 2009;18:272-281. [PubMed: 19645971]

12. Safi A, Hodgson N. Timing of activities and their effects on circadian rhythm in the elderly with dementia: A literature review. J Sleep Disord Ther 2014;3:2167-0277.

13. Brooks A, Lack L. A brief afternoon nap following nocturnal sleep restriction: Which nap duration is most recuperative? Sleep 2006;29:831-840. [PubMed: 16796222]

14. Tietzel AJ, Lack LC. The short-term benefits of brief and long naps following nocturnal sleep restriction. Sleep 2001;24:293-300. [PubMed: 11322712]

15. Tietzel AJ, Lack LC. The recuperative value of brief and ultra-brief naps on alertness and cognitive performance. J Sleep Res 2002;11:213-218. [PubMed: 12220317]

16. Tucker MA, Fishbein W. Enhancement of declarative memory performance following a daytime nap is contingent on strength of initial task acquisition. Sleep 2008;31:197-203. [PubMed: 18274266]

17. Jewett M, Wyatt J, Ritz-De Cecco A et al. Time course of sleep inertia dissipation in human performance and alertness. J Sleep Res 1999;8:1.

18. Achermann P, Werth E, Dijk D et al. Time course of sleep inertia after nighttime and daytime sleep episodes. Arch Ital Biol 1995;134:109. [PubMed: 8919196]

19. Keage HA, Banks S, Yang KL et al. What sleep characteristics predict cognitive decline in the elderly? Sleep Med 2012;13:886-892. [PubMed: 22560827]

20. Monk TH, Buysse DJ, Carrier J et al. Effects of afternoon "siesta" naps on sleep, alertness, performance, and circadian rhythms in the elderly. Sleep 2011;24:680-687.

21. Lovato N, Lack L. The effects of napping on cognitive functioning. Prog Brain Res 2010;185:155166. [PubMed: 21075238]

22. Yu P, Shi J. Aging process in China and its influences on social economy. Chinese J Geriatr 2014;33:113-115. 
23. Zhao Y, Strauss J, Yang G et al. China Health and Retirement Longitudinal Study-2011-2012 National Baseline Users' Guide Beijing: National School of Development, Peking University, 2013.

24. About CHARLS. 2013 China Health and Retirement Longitudinal Study [on-line] Available at http://charls.ccer.edu.cn/en/page/about/charls Accessed December 12, 2014.

25. Huang W, Zhou Y. Effects of education on cognition at older ages: Evidence from China's Great Famine. Soc Sci Med 2013;98:54-62. [PubMed: 24331882]

26. Lei X, Smith JP, Sun X et al. Gender differences in cognition in China and reasons for change over time: Evidence from CHARLS. J Econ Ageing 2014;4:46-55. [PubMed: 25530942]

27. Pangman VC, Sloan J, Guse L. An examination of psychometric properties of the Mini-Mental State Examination and the standardized Mini-Mental State Examination: Implications for clinical practice. Appl Nurs Res 2000;13:209-213. [PubMed: 11078787]

28. Folstein MF, Folstein SE, McHugh PR. 'Mini-mental state'. A practical method for grading the cognitive state of patients for the clinician. J Psychiatr Res 1975;12:189-198. [PubMed: 1202204]

29. McArdle JJ, Fisher GG, Kadlec KM. Latent variable analyses of age trends of cognition in the Health and Retirement Study, 1992-2004. Psychol Aging 2007;22:525. [PubMed: 17874952]

30. Mathuranath P, Nestor P, Berrios G et al. A brief cognitive test battery to differentiate Alzheimer's disease and frontotemporal dementia. Neurology 2000;55:1613-1620. [PubMed: 11113213]

31. Grandner MA, Hale L, Moore M. Mortality associated with short sleep duration: The evidence, the possible mechanisms, and the future. Sleep Med Rev 2010;14:191-203. [PubMed: 19932976]

32. Whinnery J, Jackson N, Rattanaumpawan P et al. Short and long sleep duration associated with race/ethnicity, sociodemographics, and socioeconomic position. Sleep 2014;37:601. [PubMed: 24587584]

33. Huang W, Lei X, Ridder G et al. Health, height, height shrinkage, and SES at older ages: Evidence from China. Am Econ J Appl Econ 2013;5:86-121. [PubMed: 26594311]

34. Andresen EM, Malmgren JA, Carter WB. Screening for depression in well older adults: Evaluation of a short form of the CES-D. Am J Prev Med 1994;10:77-84. [PubMed: 8037935]

35. Lei X, Sun X, Strauss J. Health outcomes and socio-economic status among the mid-aged and elderly in China: Evidence from the CHARLS national baseline data. J Econ Ageing 2014;3:29_ 43.

36. Blackwell T, Yaffe K, Ancoli-Israel S et al. Poor sleep is associated with impaired cognitive function in older women: The Study of Osteoporotic Fractures. J Gerontol A Biol Sci Med Sci 2006;61A:405-410.

37. Faubel R, Lo'pez-Garcia E, Guallar-castillon $P$ et al. Usual sleep duration and cognitive function in older adults in Spain. J Sleep Res 2009;18:427-435. [PubMed: 19691473]

38. Auyeung TW, Lee JS, Leung J et al. Cognitive deficit is associated with phase advance of sleepwake rhythm, daily napping, and prolonged sleep duration - a cross-sectional study in 2,947 community-dwelling older adults. Age (Dordr) 2013;35:479-486. [PubMed: 22215376]

39. Molloy DW, Standish TI. A guide to the standardized Mini-Mental State Examination. Int Psychogeriatr 1997;9 (Suppl 1):87-94; discussion 143-50. [PubMed: 9447431] 
A

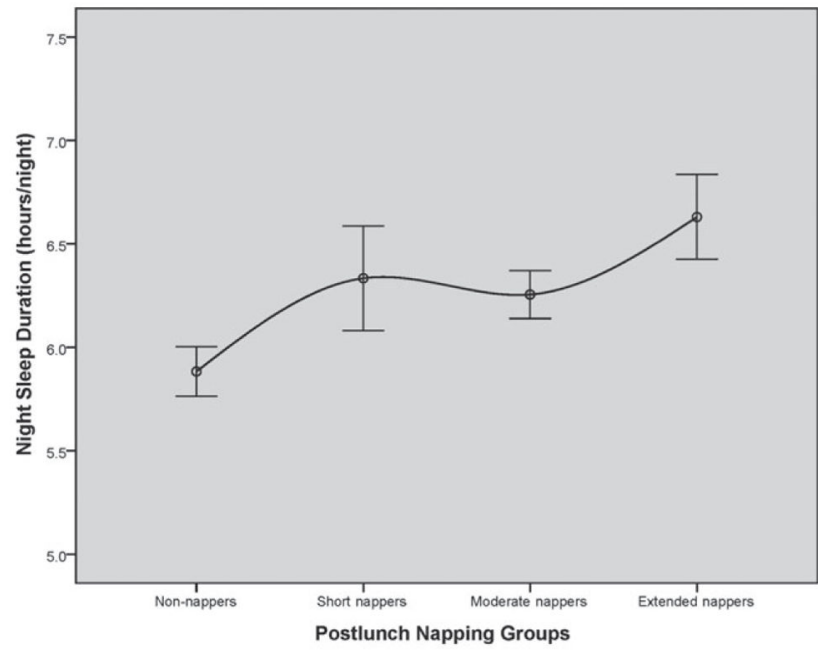

B

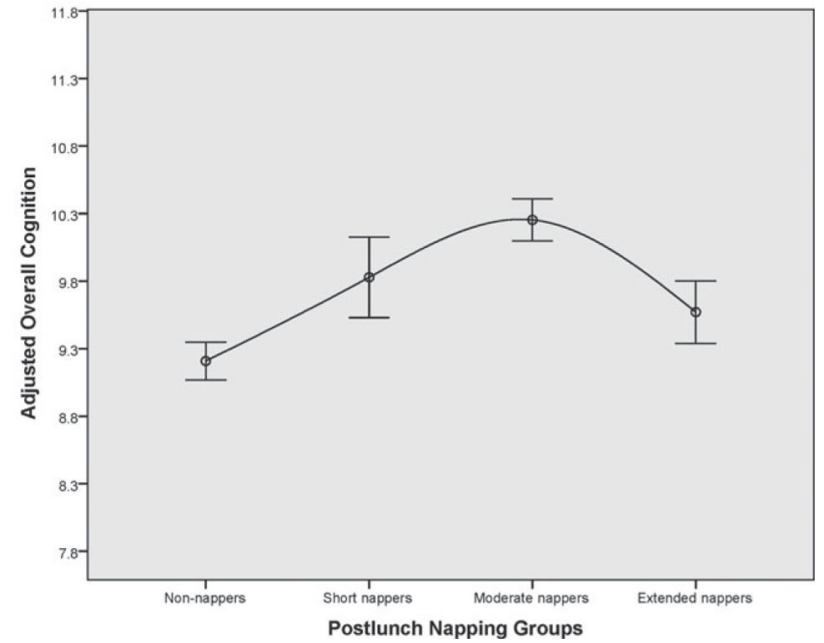

Figure 1.

(A) Association between postlunch napping ( $x$-axis) and nighttime sleep duration (hours/ night; $y$-axis). The scale of the $y$-axis represents the 25th and 75th percentiles of night sleep duration in the sample (5.0-7.5 hours/night). The black dots represent mean nighttime sleep duration, and the error bars represent $95 \%$ confidence intervals. In general, mean nighttime sleep duration increased with amount of postlunch napping. (B) Relationship between postlunch napping ( $x$-axis) and adjusted overall cognition score ( $x$-axis) from Model 3 (adjusted for age, sex, education, body mass index, depression, instrumental activities of daily living, social activities, and night sleep; $y$-axis). The scale of the $y$-axis represents the 25th and 75th percentiles of adjusted overall cognition score in the sample (7.8-11.6). The black dots represent mean adjusted overall cognition, and the error bars represent $95 \%$ confidence intervals. Overall, moderate napping was associated with higher adjusted 
cognition scores. There was also evidence of an inverted U-shaped association, such that non-nappers and extended nappers had lower adjusted overall cognition scores. 


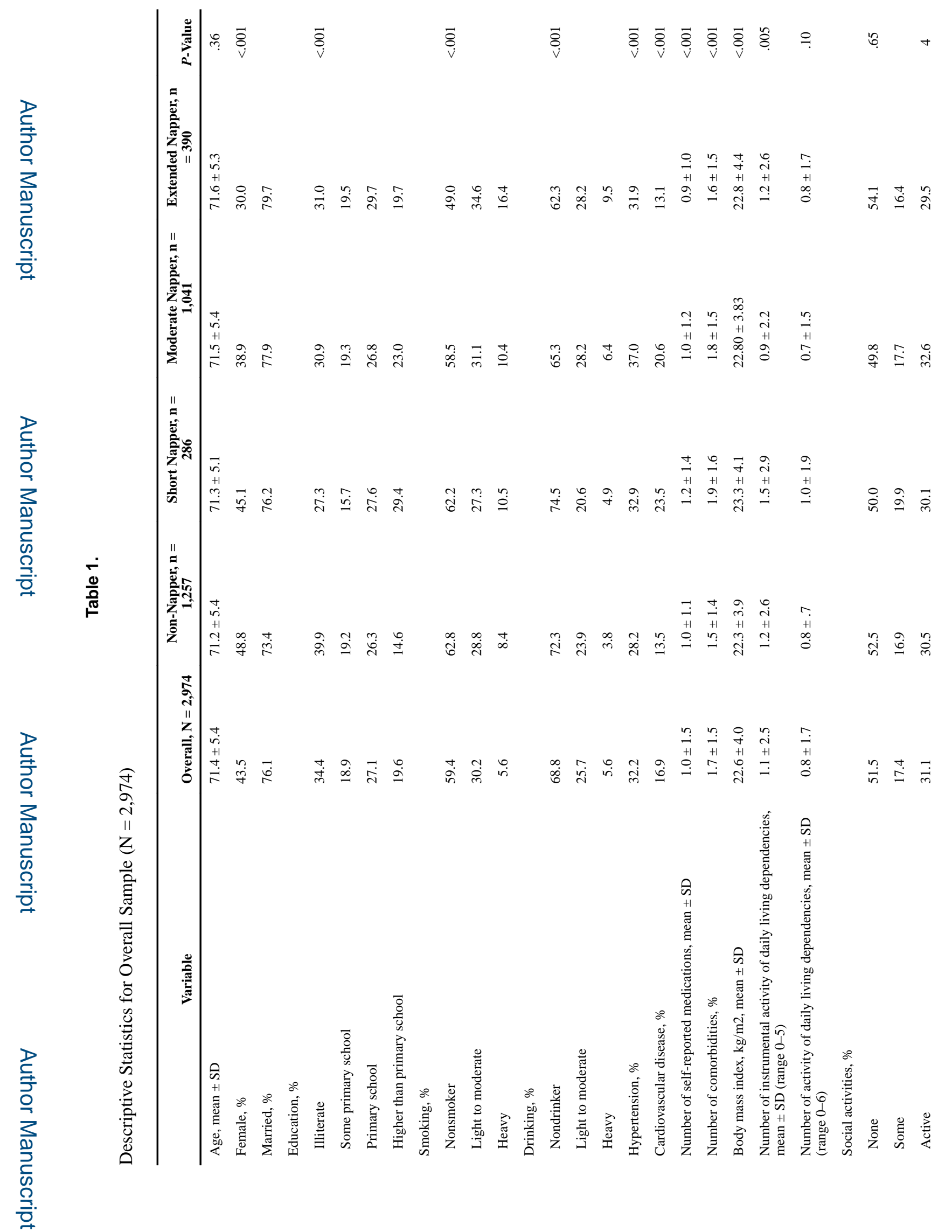

J Am Geriatr Soc. Author manuscript; available in PMC 2019 April 29. 


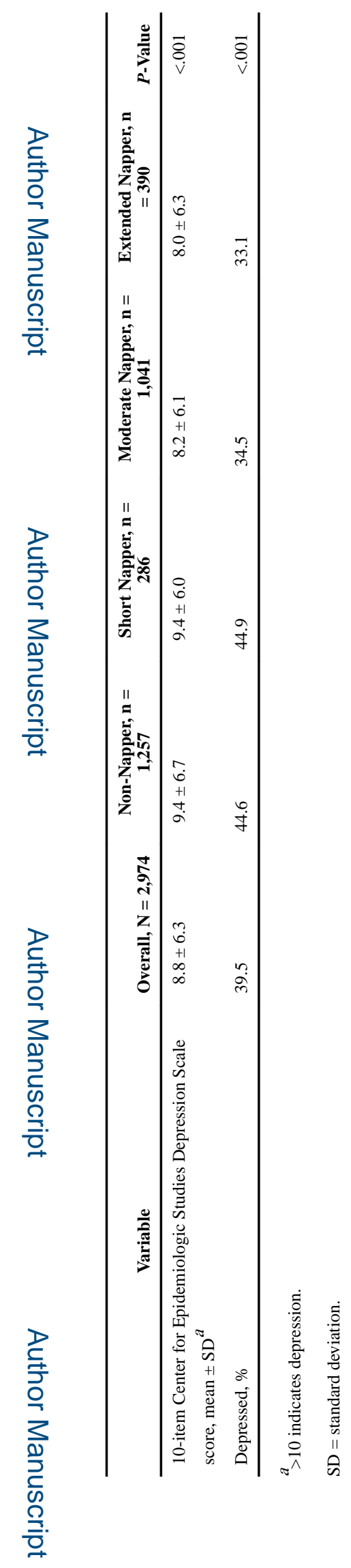




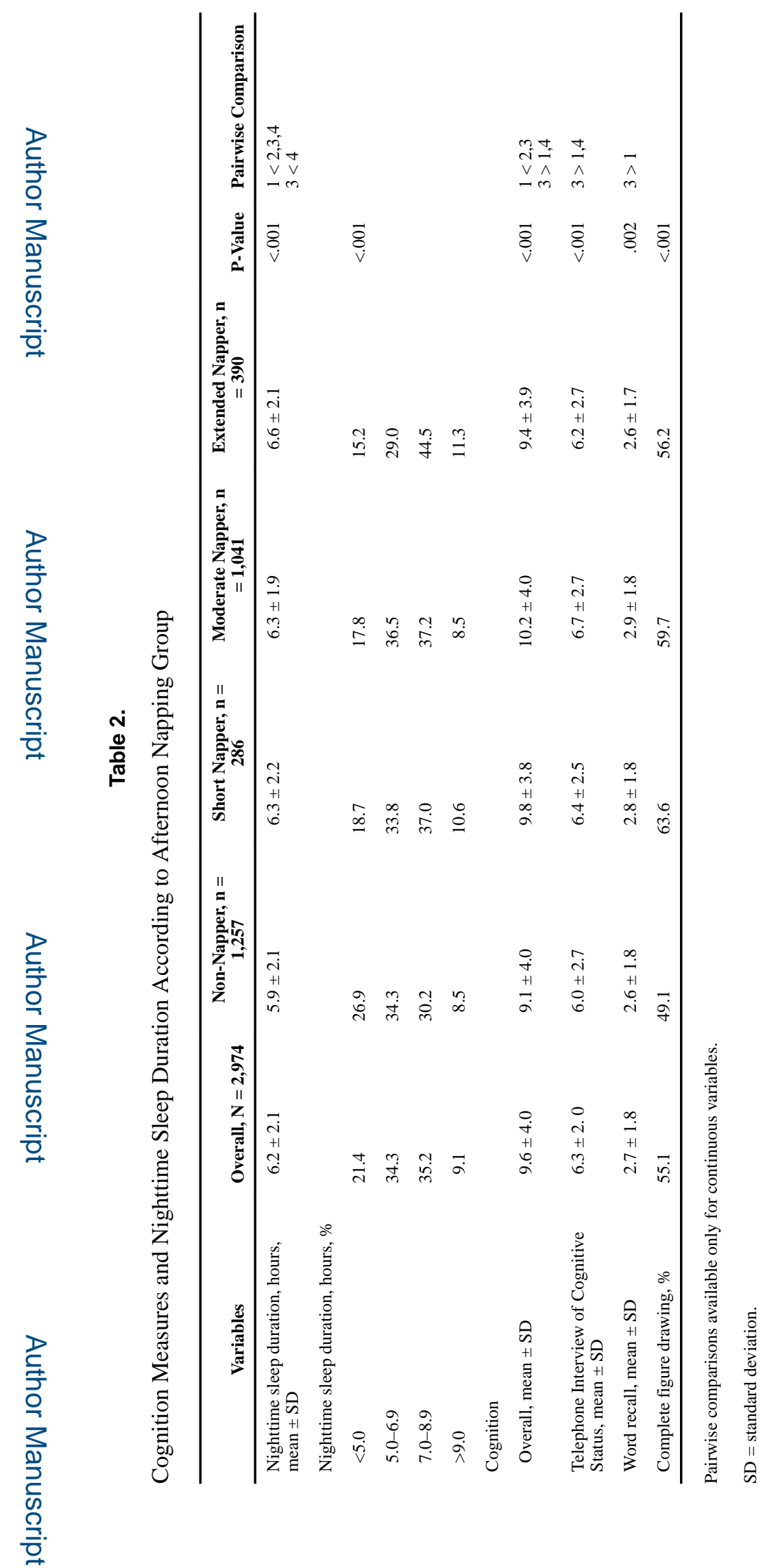

J Am Geriatr Soc. Author manuscript; available in PMC 2019 April 29. 


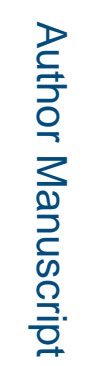

를

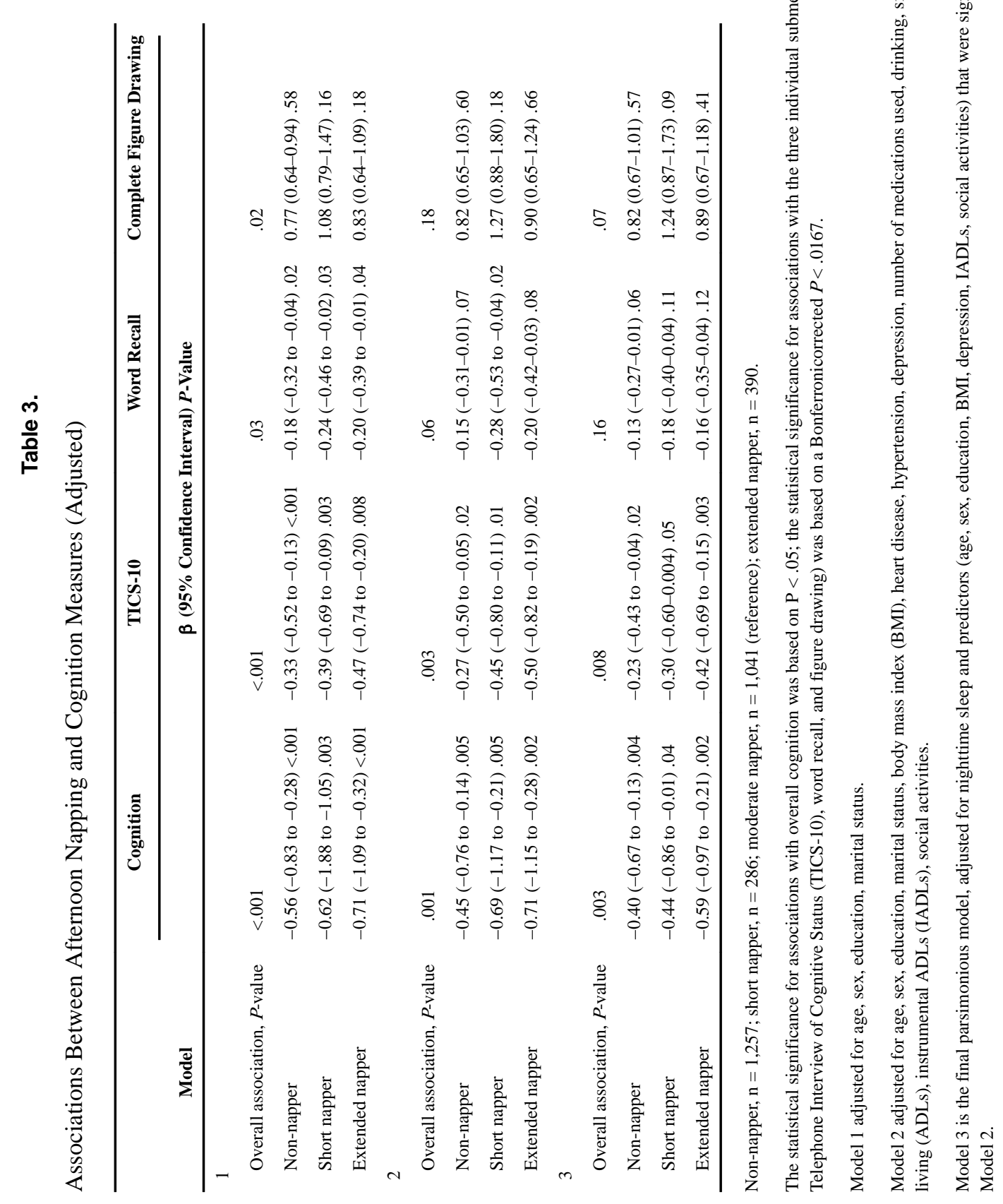

J Am Geriatr Soc. Author manuscript; available in PMC 2019 April 29. 